\title{
Age-dependent balance of leptin and ghrelin regulates bone metabolism
}

Ghrelin and leptin can promote and suppress osteoclastogenesis. These hormones interact to control bone metabolism and their relationship changes with age, according to a new study published in Endocrinology.

"We found that ghrelin regulates osteoclastogenesis and that leptin and ghrelin control bone metabolism through an interaction that changes with age," explains senior author Hans van Leeuwen from Erasmus University Medical Centre in Rotterdam, The Netherlands.

The researchers used mouse models that lack the ghrelin receptor and have leptin deficiency to investigate how these hormones influence bone metabolism and found the effects were altered with aging.

During the first 3 months of life, both hormones could stimulate osteoclastogenesis in a central or systemic manner; leptin acts through the sympathetic nervous system but the stimulatory mechanism of ghrelin is unclear. However, ghrelin also has a locally suppressive effect on osteoclast differentiation and fusion, and leptin can suppress bone resorption by stimulating hypothalamic cocaine and amphetamineregulated transcript expression-a process that can be inhibited by ghrelin.

The systemic effects of ghrelin were no longer evident at 6 months but it still exerted local suppressive effects on osteoclastogenesis. The sympathetic nervous system effects of leptin increased but could be countered by local ghrelin suppression of osteoclast formation. Deletion of the ghrelin receptor led to a decline in bone mass at 6 months, indicating a protective role for ghrelin.

The researchers are examining the interaction of these hormones and trying to establish whether the acylation status of ghrelin influences its role in bone metabolism. The age-dependent changes observed could be relevant in development of osteoporosis in elderly humans.

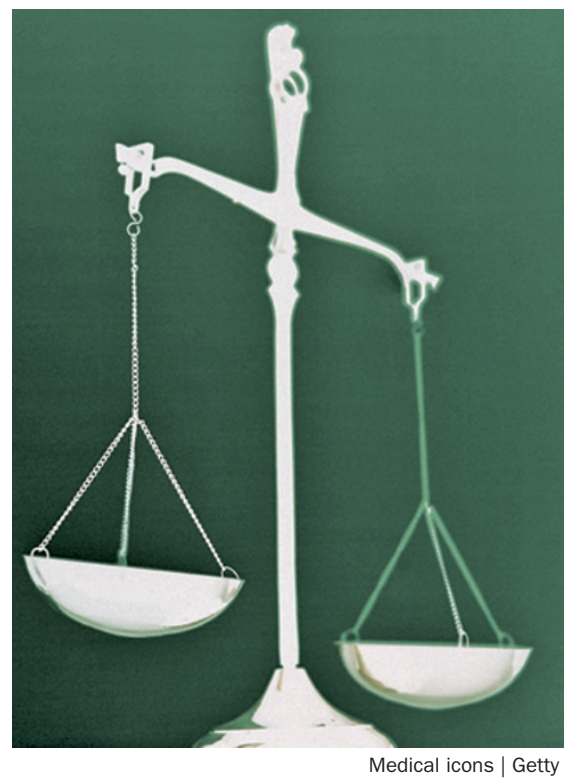

"Targeted use of ghrelin agonists could help to control age-related bone diseases," speculates van Leeuwen.

Andy McLarnon

Original article van der Velde, M. et al. An age-dependen interaction with leptin unmasks ghrelin's bone-protective effects. Endocrinology doi:10.1210/en.2012-1277 